\title{
In-vivo validation of a new clinical tool to quantify three- dimensional myocardial strain using ultrasound
}

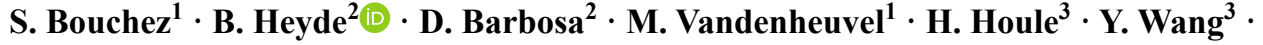 \\ J. D'hooge ${ }^{2}$ P. F. Wouters ${ }^{1}$
}

Received: 22 June 2016 / Accepted: 12 August 2016

(C) Springer Science+Business Media Dordrecht 2016

\begin{abstract}
Three-dimensional (3D) strain analysis based on real-time 3-D echocardiography (RT3DE) has emerged as a novel technique to quantify regional myocardial function. The goal of this study was to evaluate accuracy of a novel model-based 3D tracking tool (eSie Volume Mechanics, Siemens Ultrasound, Mountain View, CA, USA) using sonomicrometry as an independent measure of cardiac deformation. Thirteen sheep were instrumented with microcrystals sutured to the epi- and endocardium of the inferolateral left ventricular wall to trace myocardial deformation along its three directional axes of motion. Paired acquisitions of RT3DE and sonomicrometry were made at baseline, during inotropic modulation and during myocardial ischemia. Accuracy of 3D strain measurements was quantified and expressed as level of agreement with sonomicrometry using linear regression and Bland-Altman analysis. Correlations between 3D strain analysis and sonomicrometry were good for longitudinal and circumferential strain components $(r=0.78$ and $r=0.71)$ but poor for radial strain $(\mathrm{r}=0.30)$. Accordingly, agreement (bias $\pm 2 \mathrm{SD})$ was $-5 \pm 6 \%$ for longitudinal, $-5 \pm 7 \%$ for circumferential, and $15 \pm 19 \%$ for radial strain. Intra-observer variability was low for all components (intra-class correlation coefficients (ICC) of respectively 0.89, 0.88 and 0.95) while
\end{abstract}

S. Bouchez

stefaan.bouchez@ugent.be

1 Department of Anesthesiology, Ghent University Hospital, De Pintelaan 185, 9000 Ghent, Belgium

2 Laboratory on Cardiovascular Imaging and Dynamics, KU Leuven, Leuven, Belgium

3 Ultrasound Division, Siemens Medical Solutions, Mountain View, CA, USA inter-observer variability was higher, in particular for radial strain $(\mathrm{ICC}=0.41)$. The present study shows that $3 \mathrm{D}$ strain analysis provided good estimates of circumferential and longitudinal strain, while estimates of radial strain were less accurate between observers.

Keywords Cardiology - Ultrasound imaging · Ventricular mechanics · In-vivo validation · Sonomicrometry $\cdot$ Strain

\section{Introduction}

Strain analysis is increasingly being advocated as an objective method to quantify global and regional myocardial deformation [1-5]. Commercially available tools for 2D strain analysis show reasonable accuracy in the experimental setting and have definitely entered clinical practice. However, as myocardial deformation occurs in a three dimensional (3D) field, 2D analysis can only partially describe this complex phenomenon and may include errors due to out-of-plane motion of tissue under investigation. Furthermore, a complete topographic analysis of all strain components in the left ventricle (LV) requires a minimum of six separate image acquisitions.

3D strain analysis based on real-time 3D echocardiography (RT3DE) is expected to overcome these limitations and provide a more practical and complete approach to global and regional ventricular dynamics [6, 7]. With full volume acquisition, RT3DE incorporates all strain components of the heart within a single dataset [8-10]. In addition, $3 \mathrm{D}$ strain analysis is angle independent and avoids strain estimation errors due to out-of-plane motion. Finally, it provides new indices such as area strain and may enable more precise calculations of LV twist and shear strain. 
Despite these obvious conceptual advantages, the clinical utility of 3D strain analysis remains to be demonstrated. Indeed, the increased field of view of volumetric images limits the spatial and temporal resolution of the dataset. Current volumetric datasets therefore have a relatively coarse speckle pattern that exhibits large amounts of motion and deformation between subsequent volumes. These conditions increase the risk of speckle decorrelation and tracking errors.

To date, several software tools, based on distinct principles and algorithms, are available for 3D tracking and a number of clinical studies have shown their potential to assess regional function [11-13]. However, few studies have experimentally validated these methods in an in-vivo setting [13] and results seem to vary between the different analytic approaches $[14,15]$. In this study we evaluate a newly developed model-based software package for 3D strain analysis. Our working hypothesis is that there is good agreement for quantitative values of all three strain components assessed by RT3DE-based 3D strain analysis with the current gold standard technique, i.e. invasive sonomicrometry. We addressed this hypothesis in an acutely instrumented open-chest sheep model subjected to inotropic modulation and controlled regional myocardial ischemia.

\section{Methods}

\section{Animals}

This study was performed in thirteen female Suffolk sheep (mean body weight, $44 \pm 11 \mathrm{~kg}$ ). The study conformed with the Public Health Service Policy on Humane Care and Use of Laboratory Animals published by the Office of Laboratory Animal Welfare of the United States National Institutes of Health [16] and was approved by the local ethics committee (Ethische Commissie Dierproeven, Ghent University, Ghent, Belgium).

\section{Instrumentation}

The sheep were premedicated with ketamine $(10 \mathrm{mg} / \mathrm{kg})$ and piritramide $(1 \mathrm{mg} / \mathrm{kg})$. Anesthesia was induced with sufentanil $(0.5 \mu \mathrm{g} / \mathrm{kg})$ and an intravenous infusion of propofol $(10 \mathrm{mg} / \mathrm{kg})$. The trachea was intubated and the lungs were mechanically ventilated throughout the procedure with a mixture of sevoflurane, oxygen, and room air to maintain normal blood oxygen and carbon dioxide levels (tidal volume, $8 \mathrm{~mL} / \mathrm{kg}$; respiratory rate, 12 breaths $/ \mathrm{min}$ ). A gastric tube was positioned to evacuate excess gas and fluid from the reticulorumen. Anesthesia was maintained with a continuous infusion of sufentanil $(1 \mu \mathrm{g} / \mathrm{kg} / \mathrm{h})$ and sevoflurane inhalation (end-tidal concentration, $2.5 \%$ ).
A bilumen catheter was inserted into the left jugular vein to allow measurement of central venous pressure and administration of drugs. A catheter-tipped pressure transducer (Millar, Houston, TX, USA) was advanced into the LV chamber via the right carotid artery for continuous monitoring of LV pressure and first temporal derivative $(\mathrm{dP} / \mathrm{dt})$. The systemic arterial pressure was measured in the proximal aorta using a fluid-filled sideline of the arterial sheet.

Cisatracurium (bolus, $0.5 \mathrm{mg} / \mathrm{kg}$ ) was administered $10 \mathrm{~min}$ before the surgical incision. A sternotomy was performed, and the heart was suspended in a pericardial cradle to maintain normal anatomic configuration. Cardiac output was monitored with a flow probe positioned around the pulmonary artery.

Ventricular wall deformation throughout the cardiac cycle was determined with sonomicrometry (Sonometrics Ultrasonic Measurement System, Sonometrics Corporation, London, Ontario, Canada). Reference radial $\left(\varepsilon_{\mathrm{RR}}\right)$, longitudinal $\left(\varepsilon_{\mathrm{LL}}\right)$, and circumferential $\left(\varepsilon_{\mathrm{CC}}\right)$ strain components were determined using four ultrasonic microcrystals that were sutured in a tetrahedral configuration to the myocardium at the mid-inferolateral wall; three crystals were sutured to the epicardium, resulting in two crystal pairs along the circumferential (parallel to the AV-groove) and longitudinal direction (perpendicular to the AV groove), and one crystal was placed in the subendocardial region just radial to the center crystal. The subendocardial crystal was introduced obliquely to limit damage to the evaluated myocardium (Fig. 1).

\section{Data acquisition}

Full-volume, real-time ultrasonographic volumetric data (RT3DE) were recorded with an ultrasound machine (Siemens SC2000, Siemens Ultrasound, Mountain View, CA, USA) equipped with a matrix transducer (4Z1c matrix transducer, Siemens). Volume sequences were acquired from an apical window using a liver as standoff. The optimal combination of spatial and temporal resolution was achieved by decreasing the volume size and depth to the smallest setting possible, while keeping the whole LV chamber within the field of view. The ultrasound transducer operated at a central frequency of $2.8 \mathrm{MHz}$ and acquired images from the entire heart at 25-50 volumes per second.

After echocardiographic recordings at baseline, the inotropic state was reduced by esmolol infusion and increased by administration of dobutamine. This enabled modulation of the range of strain values. A physiologic target was set to $50 \%$ decrease in $\mathrm{LV} \mathrm{dP} / \mathrm{dt}$ with esmolol, and $100 \%$ increase in $\mathrm{LV} \mathrm{dP/dt}$ with dobutamine titration, with respect to baseline. Finally, acute ischemia was induced by snare occlusion of the circumflex coronary artery.

The sonomicrometry and RT3DE systems could not be operated simultaneously because the systems use 

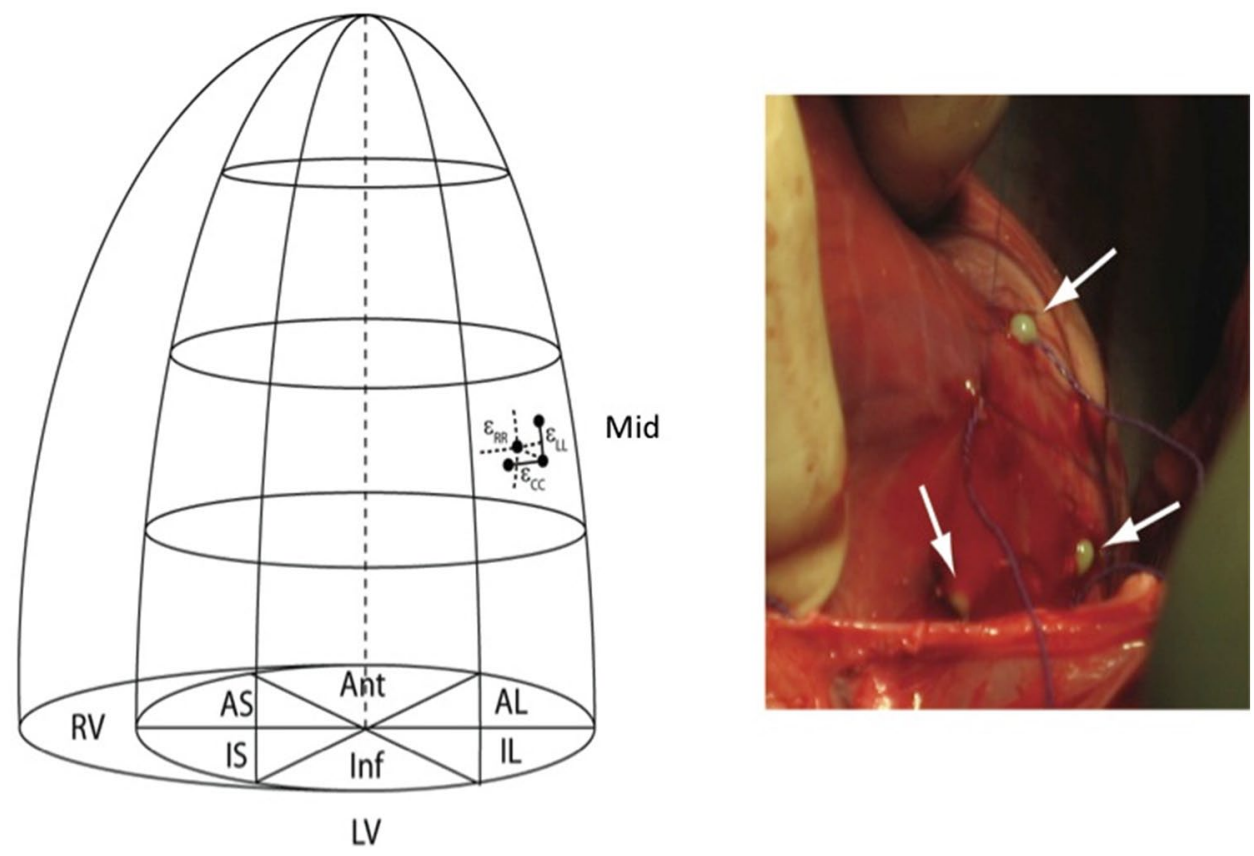

$\left(\varepsilon_{\mathrm{LL}}\right)$, and circumferential $\left(\varepsilon_{\mathrm{CC}}\right)$ direction. $A L$ anterolateral, Ant ante-

Fig. 1 Determination of ventricular wall deformation with sonomicrometry. Sonomicrometry crystals (white arrows) were sutured in a tetrahedral configuration in the inferolateral cardiac wall to obtain reference myocardial deformation $(\varepsilon)$ in the radial $\left(\varepsilon_{\mathrm{RR}}\right)$, longitudinal

overlapping frequency bands. Therefore, crystal data were acquired immediately before and after each stage, and the sonomicrometry system was switched off during the RT3DE recordings made in between.

\section{Determination of myocardial strain}

All 3D datasets were processed using a software package for automated volumetric strain analysis (eSie Volume Mechanics, Siemens Healthcare, Erlangen, Germany) that allowed extraction of segmental curves for $\varepsilon_{\mathrm{RR}}, \varepsilon_{\mathrm{LL}}$, and $\varepsilon_{\mathrm{CC}}$. Four $2 \mathrm{D}$ reference planes were automatically generated from the acquired 3D volumetric datasets and positioned at the enddiastolic frame. The software features an automatic LV segmentation solution, but this was designed for human hearts. It was therefore not appropriate to analyze sheep cardiac images. As such, a semi-automatic segmentation strategy was followed to delineate the region of interest for analysis. In each of the four views, an endocardial border was first constructed from three reference points that were placed at the level of the mitral annulus and one reference point that was placed at the LV apex. The epicardial border was then set at a fixed distance from the endocardial contour. Finally, endo- and epicardial borders could be manually adjusted and the resulting myocardial mesh was tracked throughout the cardiac cycle. The three cardiac strain components were estimated in a segmented LV model (Fig. 2). The end-systolic frame was determined visually based on aortic valve closure. rior, $A S$ anteroseptal, $I L$ inferolateral, Inf inferior, $I S$ inferoseptal, $L V$ left ventricle, $M i d$ mid-ventricular, $R V$ right ventricle

Reference strain curves were obtained from processing the recorded crystal traces with custom software as described previously [17]. By using the speed of sound $(1530 \mathrm{~m} / \mathrm{s})$ and the time of flight between ultrasonographic emission and detection in a neighboring crystal, inter-crystal distance was calculated continuously (time resolution, $1 \mathrm{~ms}$; spatial resolution, $15.4 \mu \mathrm{m}$ ). End-diastole for the crystal traces was identified from the onset of the LV contraction at the positive infliction point of the first LV pressure derivative. Strain was calculated using [4]:

$\varepsilon_{N}(t)=\frac{D_{N}(t)-D_{N}(0)}{D_{N}(0)}$

where $D_{N}(t)$ was the instantaneous distance at time point $t$ between two crystals in direction $\mathrm{N}$ (radial, circumferential, or longitudinal) and $\mathrm{D}_{\mathrm{N}}(0)$ was the distance in direction $\mathrm{N}$ at time zero, which corresponded to end-diastole. End-systolic values were extracted from the time of aortic valve closure, defined as $\mathrm{dP} / \mathrm{dt}_{\min }-20 \mathrm{~ms}$ [18]. The reference end-systolic strain values before and after each stage were averaged to account for any physiologic change during the time of ultrasound acquisitions.

\section{Pairing of crystal and 3D strain measurements}

Although the sonomicrometry crystals were implanted in the inferolateral wall at the mid-ventricular level, there 


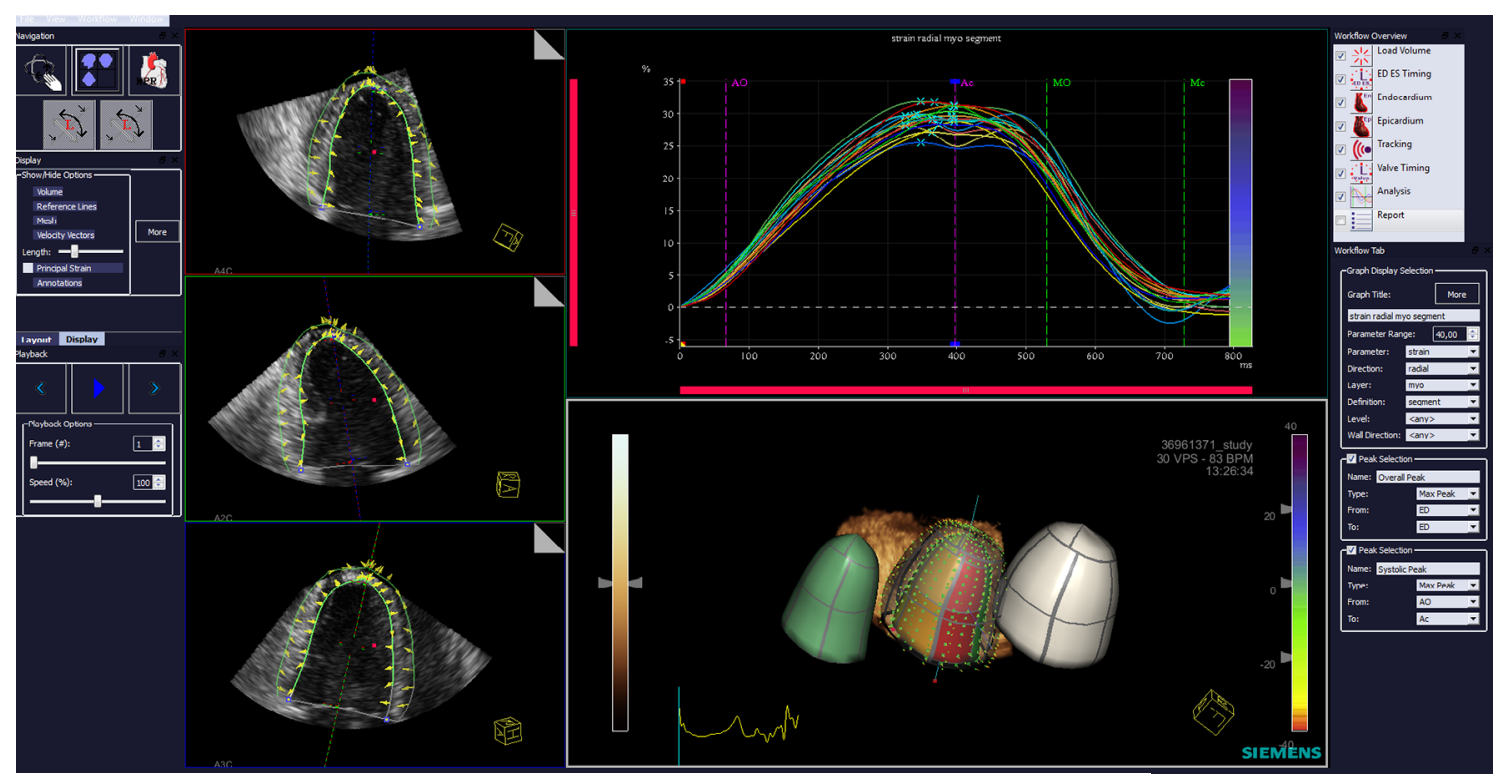

Fig. 2 Display of segmental radial strain values after tracking the endocardial and epicardial borders

was uncertainty in matching the anatomic location to the 3D mesh extracted from the volumetric ultrasound data because the sonomicrometry crystals were not visible in the ultrasound volumes. Although this was of benefit to avoid bias induced by tracking the crystals instead of the myocardium, it did pose the problem of spatial registration between the crystal and echocardiographic strain measurements. As such, the segment defined as the mid-ventricular inferolateral segment in the 3D echocardiographic mesh may not exactly correspond to the anatomic position of the implanted crystals. While this issue is less critical for a normal heart showing a fairly homogeneous wall deformation, this becomes important in an ischemic scenario because major spatial differences in regional strain occur, even for anatomically adjacent regions. To avoid this potential error, we used an iterative process, fitting the wider inferolateral area, including segment 11 and its direct anatomic neighbors (segments 5-10-12-16) to the sonomicrometry data of all experimental stages per individual animal. The segment numbers refer to the standardized AHA segment definition [19]. The best fitting segment for a given animal was then selected and consistently used as the index sample for data analysis over all experimental stages in that particular animal.

\section{Statistical analysis}

Statistical analysis was performed with two software packages (MATLAB R2010b, MathWorks, Natick, MA, USA and Excel 2007, Microsoft Corp., Redmond, WA, USA). Assessment of accuracy and agreement between the reference local end-systolic strain measured by sonomicrometry and RT3DE was performed for each strain component with linear regression and Bland-Altman analysis. This analysis was also performed per hemodynamic stage (baseline, dobutamine, esmolol, ischemia), to investigate whether the performance of the clinical strain tool was affected by the heart rate. Bias and limits of agreement differences between stages were assessed using a $t$ test and F-test respectively. Two assessors analyzed the datasets independently in order to study inter-observer variability. One of the assessors performed the complete analysis twice after a 1-week interval to determine intra-observer variability. Inter- and intraobserver variability were quantified and reported using the intra-class correlation coefficient (ICC). Statistical significance was defined as $P \leq 0.05$.

\section{Results}

Ischemia measurements could not be completed in four sheep due to major arrhythmia and esmolol data was lacking in one animal. Three ultrasound datasets were excluded from analysis because of image dropout artifacts in the inferolateral region ( 1 dataset during esmolol and 2 datasets during ischemia). Reference sonomicrometry strain values were not available at baseline for seven sheep because of missing pressure traces, which prevented reliable timing of end-systole. In addition, reference strain traces of two animals in the ischemic phase were excluded because of unreliable signal quality from the sonomicrometry system. Overall, 35 datasets were thus included for further analysis by 2 independent users. Hemodynamic data are shown in Table 1.

3D strain analysis and sonomicrometry data correlated well for longitudinal and circumferential strain components 
$(r=0.78$ and $r=0.71)$ but not for radial strain $(r=0.30)$. Correlations between average echo strain and crystals in the setting without ischemia were $r=0.77$ for longitudinal, $r=0.50$ for circumferential and $r=0.08$ for radial strain respectively. 3D strain measurements were consistently higher than sonomicrometric measurements for all components but the magnitude of difference was highest for radial strain. Limits of agreement were also two- to threefold higher for radial strain compared to longitudinal and circumferential strain (Fig. 3). Table 2 summarizes bias and limits of agreement for all stages or grouped per investigated hemodynamic stage.

Intra-observer variability was low for all strain components (Table 3). Inter-observer variability was moderate for

Table 1 Hemodynamics during the 4 acquisition stages

\begin{tabular}{lcllll}
\hline & HR (bpm) & EDV $(\mathrm{ml})$ & $\begin{array}{l}\text { ESV } \\
(\mathrm{ml})\end{array}$ & EF (\%) & $\begin{array}{l}\text { VR } \\
(\mathrm{V} / \mathrm{s})\end{array}$ \\
\hline Baseline & $85 \pm 8$ & $74 \pm 19$ & $32 \pm 12$ & $58 \pm 8$ & $35 \pm 11$ \\
Dobutamine & $123 \pm 14^{*}$ & $58 \pm 16^{*}$ & $28 \pm 9$ & $53 \pm 5$ & $35 \pm 11$ \\
Esmolol & $72 \pm 6^{*}$ & $79 \pm 20$ & $36 \pm 10$ & $55 \pm 5$ & $36 \pm 11$ \\
Ischemia & $101 \pm 16$ & $74 \pm 27$ & $44 \pm 23$ & $44 \pm 15$ & $34 \pm 12$ \\
\hline
\end{tabular}

$H R$ heart rate, $E D V$ end diastolic volume, $E S V$ end systolic volume, $E F$ ejection fraction, $V R$ volume rate

$* P \leq 0.05$ longitudinal and circumferential strain ICC of 0.67 and 0.60 respectively but high for radial strain (ICC 0.41).

\section{Discussion}

The present data show good correlation and agreement between RT3DE and sonomicrometry for quantification of regional longitudinal and circumferential strain, but not for the radial component. Inter-observer variability was also highest for radial strain, suggesting that these calculations

Table 2 Bland-Altman analysis of end-systolic strain determined from RT3DE and sonomicrometry for radial, longitudinal and circumferential strain

\begin{tabular}{llll}
\hline \multirow{2}{*}{} & \multicolumn{3}{l}{$\begin{array}{l}\text { Bland-Altman analysis (bias } \pm \text { limits of } \\
\text { agreement) }\end{array}$} \\
\cline { 2 - 4 } & Radial & Longitudinal & Circumferential \\
\hline Global & $15.1 \pm 19.0$ & $-4.9 \pm 6.5$ & $-5.4 \pm 7.4$ \\
Baseline & $18.8 \pm 21.4$ & $-6.8 \pm 5.7$ & $-5.7 \pm 7.3$ \\
Dobutamine & $11.4 \pm 20.0$ & $-4.5 \pm 5.9$ & $-3.5 \pm 7.4$ \\
Esmolol & $16.7 \pm 18.7$ & $-4.8 \pm 8.2$ & $-7.0 \pm 6.5$ \\
Ischemia & $15.8 \pm 13.3$ & $-3.8 \pm 3.5$ & $-5.8 \pm 7.4$ \\
\hline
\end{tabular}

Analysis was performed globally, or when grouping datasets per stage. Limits of agreement was as $1.96 \times \mathrm{SD}$
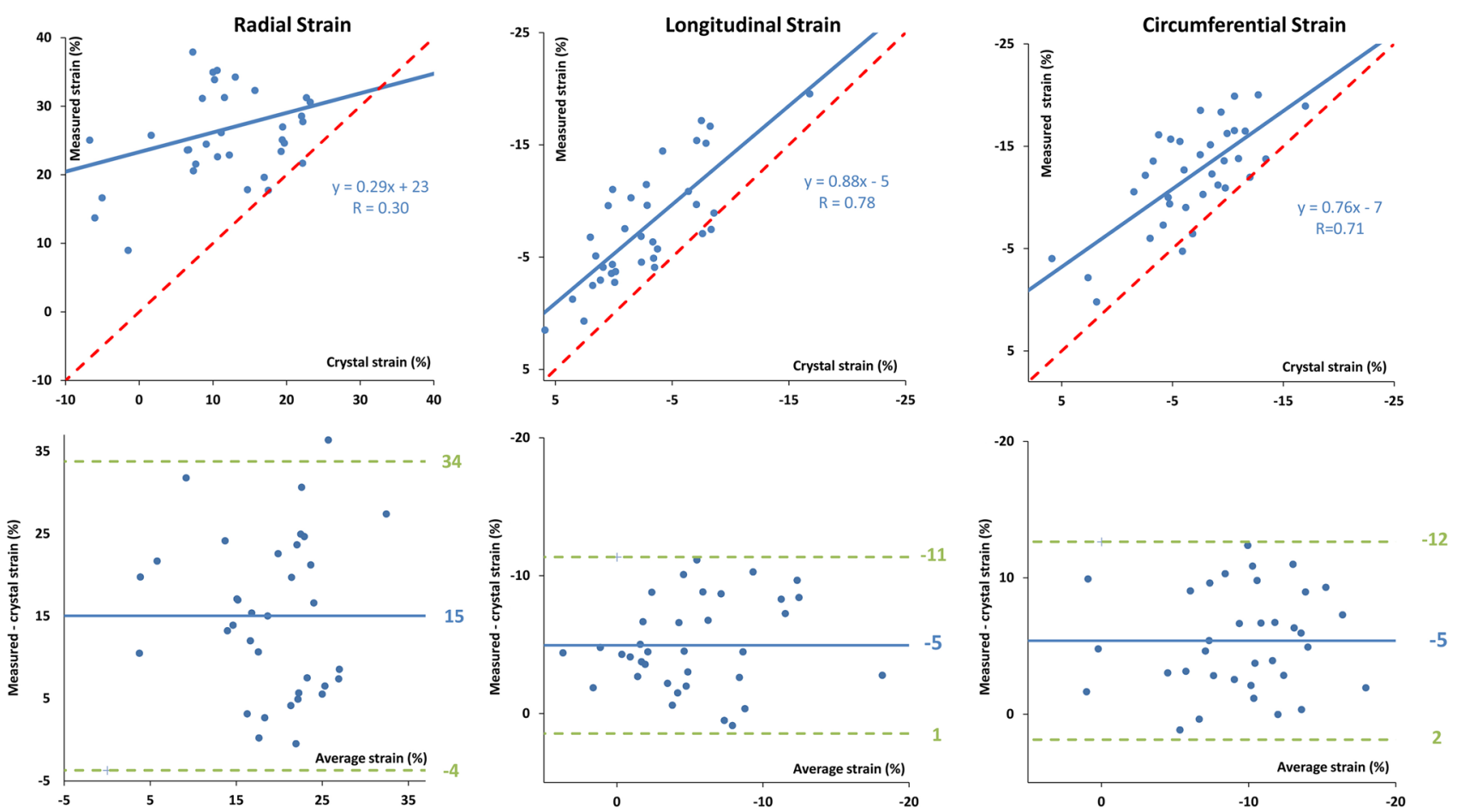

Fig. 3 Correlation analysis and Bland-Altman plots (bias and limits of agreement) of end-systolic strain determined from RT3DE and sonomicrometry for radial (left), longitudinal (middle) and circumferential strain (right) 
Table 3 Intra- and interobserver variability expressed as intra-class coefficient (ICC) for strain analysis with real-time 3-Dimensional echocardiography

\begin{tabular}{llll}
\hline & \multicolumn{2}{l}{ Strain component } \\
\cline { 2 - 4 } & Radial & Longitudinal & Circumferential \\
\hline Intraobserver ICC & 0.95 & 0.89 & 0.88 \\
Interobserver ICC & 0.41 & 0.67 & 0.60 \\
\hline
\end{tabular}

are user-dependent and subject to interpretation. This study thus presents an in-vivo validation of a new model-based algorithm for 3D strain analysis. The results confirm that 3D strain analysis has the potential to allow rapid and complete assessment of regional ventricular dynamics.

These findings are consistent with results obtained from current commercial 3D speckle tracking methods that rely on block-matching-based algorithms. The superiority of RT3DE over previously used 2D techniques in terms of assessing global myocardial function, has already been suggested [20], but only a few studies have validated these 3D algorithms in an in-vivo experimental setting. Regional correlations between 3D speckle tracking imaging (3D-STI) and sonomicrometry were previously reported by Seo et al. in ten sheep $(\varepsilon R R, r=0.59-0.70 ; \varepsilon L L, r=0.65-0.68 ; \varepsilon C C$, $\mathrm{r}=0.71-0.78$; [12]). In a clinical study [21], 3D strain values were compared to $2 \mathrm{D}$ techniques, and segmental correlations were moderate for $\varepsilon \mathrm{LL}(\mathrm{r}=0.49)$ and $\varepsilon \mathrm{CC}(\mathrm{r}=0.43)$ but poor for $\varepsilon R R(r=0.24)$. In [17], we described an alternative tracking technique based on elastic image registration. In eleven sheep, a duplicate set of echocardiographic recordings was made at each experimental stage with a distinct echocardiography system. All strain components were estimated with acceptable accuracy $(\varepsilon R R, r=0.69$; $\varepsilon L L, r=0.64 ; \varepsilon C C, r=0.62$ ). However, caution is advised in interpreting and comparing previous results between these studies because the reported strain values may not correspond to the same local cardiac coordinate system. Indeed, in some studies principal strain components were measured along the three major directions where no shear strain occurred [22].

The lower accuracy for radial strain may relate to the fact that the spatial motion gradient has to be calculated over a smaller region. Furthermore, beam density and spatial resolution are also lower in the radial direction compared to the longitudinal direction because of the left ventricular orientation in the image. Although recording data from a parasternal transducer position might partially overcome this problem, this does not allow visualization of the whole LV in a single acquisition thereby reducing the benefit of using RT3DE over 2D techniques. Worth mentioning is that the measurement of $\varepsilon_{R R}$ is also more difficult and susceptible to errors with 2D techniques [23]. To overcome this problem, some systems have proposed to use additional parameters such as area strain (GE Healthcare, Horten, Norway) or area change ratio (Toshiba, Tokyo, Japan). These parameters combine information about the deformation occurring in the circumferential and longitudinal directions by measuring the relative area change of the endocardial surface during the cardiac cycle. The volume conservation theory notes that the sum of all strain components equals 0 for small deformations, and area strain or area change ratio may provide information similar to $\varepsilon_{R R}$ without measuring $\varepsilon_{R R}$. Indeed, an in-vivo validation study showed that area change ratio could detect myocardial ischemia as well as standard strain components and as such seems to be a promising tool to assess regional wall motion [24].

Longitudinal and circumferential strain measurements were more consistent than radial strain but also showed a bias towards overestimation of the corresponding sonomicrometry-based data (Table 2-global). Since sonomicrometric crystals were sutured to the epicardium, while RT3DE-based measurements include a significant part of the midmyocardium, this bias could at least partially relate to the transmural gradient in deformation. Implantation of the crystals in the midmyocardium however may cause considerable muscle damage and can affect speckle tracking of the myocardium with echocardiography. Interestingly, in previous studies on 2D strain analysis, no large bias was reported for longitudinal strain in spite of the same methodological issue [25].

Subgroup analysis per hemodynamic stage (Table 2) revealed that performance did not change over the considered stages: neither the bias, nor the limits of agreement of the dobutamine, esmolol and ischemia stage showed significant differences $(P<0.05)$ with respect to the baseline stage. Indeed, datasets with more frames per cardiac cycle (i.e. during esmolol) or with less frames per cardiac cycle (i.e. during dobutamine) showed the same overall performance trends. These results are in line with a recent study for $2 \mathrm{D}$ tracking software which showed that the effect of frame rate on performance becomes minimal once a sufficient frame rate is reached [26]. The findings of our study therefore seem to indicate that a sufficient volume rate was reached to reliably analyze the data in each stage. It should also be noted that the ischemia stage had a tendency to perform better for the radial and longitudinal strain component (with the lower bias in the longitudinal direction almost being statistically significant, $P=0.08$ ). This is likely related to the fact that overall deformation was also lower, potentially making tracking easier as inter-frame motion was smaller.

This study focused on accuracy of a single 3D strain analysis method and was not designed to compare different $3 \mathrm{D}$ algorithms nor to compare $2 \mathrm{D}$ with $3 \mathrm{D}$ strain analysis systems. There is certainly a need for such comparative studies in the near future. There is high variation between values obtained by software from different commercially available systems to assess 3D strain [27]. The different 
methods to measure 3D strain of the heart typically use different regularization choices or post-processing steps [27]. For that reason, it is important that every method is validated before being introduced into clinical practice. Furthermore, it is difficult to validate and compare different $3 \mathrm{D}$ strain methods because no standardization or benchmark data is available at present. Standardization should indeed become a priority for future studies addressing the clinical utility of 2D- as well as 3D strain analysis. In this context, the first steps towards 2D strain standardization have recently been set in motion by the release of consensus document regarding strain definitions [28].

\section{Study limitations}

The 3D strain assessment tool investigated in this study relies on a database developed for human data [29]. While analyzing RT3DE data, the software integrates a priori knowledge regarding relevant human cardiac motion patterns learned during the initial off-line training phase of the algorithm. It is possible that species-related differences in cardiac morphology and function were a source of error. However, validation studies in human subjects are complicated by the lack of a solid reference measurement since existing strain imaging benchmarks have their own intrinsic pitfalls. The use of a large animal model and accurate invasive measurements of local deformation still provides an adequate trade-off between a clinically realistic setting and an accompanying solid reference method.

A related problem was the need for manual correction of the initially segmented myocardial region of interest. This undoubtedly contributed to the high inter-observer variability for the radial strain component and may also have affected the correlation between strain and sonomicrometry measurements. The use of the embedded automated workflow to analyze human echocardiograms may reduce this source of error in the clinical setting. On the other hand, echocardiographic images were acquired in an open chest animal preparation, which resulted in a more consistent and better image quality than obtained on average with transthoracic imaging. Scanner settings were optimized to obtain frame rates between 30 and $50 \mathrm{~Hz}$. It is important to notice that the same frame rate can be obtained in closed chest conditions with the specific ultrasound system evaluated in this study because it uses massive parallel receive beam forming. This frame rate was previously shown to qualify for the measurement of peak strain [30].

Sonomicrometric and 3D echocardiographic measurements could not be performed simultaneously because of signal interference [31, 32]. To our opinion, this limitation had little effect on the results since paired measurements were always completed within 3 min and only during steady state conditions.
Finally, misalignment of the implanted sonomicrometric crystals is of concern, as positioning of these crystals is critical and technically difficult on the beating heart. However, trigonometric calculations of the magnitude of error induced by a potential misalignment show the following: assuming a myocardial thickness of $10 \mathrm{~mm}$ and a pure radial strain of $30 \%$, a relative error not exceeding $10 \%$ (i.e. an absolute strain error $<3 \%$ ) would require a 14 degree misalignment in both azimuth and elevation direction, or a misalignment of 19.5 degrees when only a single plane is considered. Such misalignment would be readily picked up in our 2D echocardiographic control procedure and, as such, did not occur in this study.

\section{Conclusions}

In summary, the present study shows that RT3DE with a new clinical tool (eSie Volume Mechanics, Siemens Healthcare) that combines 3D speckle tracking methods with a model-based algorithm, provides accurate estimates of $\varepsilon_{\mathrm{CC}}$ and $\varepsilon_{\mathrm{LL}}$ from volumetric ultrasonographic datasets in an open-chest animal model. Estimates of $\varepsilon_{\mathrm{RR}}$ are however less reliable.

Funding The work of B. Heyde was funded by the FWO-Flanders Research Fund under Grant G.0693.09.

\section{Compliance with ethical standards}

Conflict of interest H. Houle and Y. Wang are part of the Ultrasound Division of Siemens Medical Solutions. The other authors declare they have no conflict of interest.

Ethical approval This study conformed with the Public Health Service Policy on Humane Care and Use of Laboratory Animals published by the Office of Laboratory Animal Welfare of the United States National Institutes of Health [16] and was approved by the local ethics committee (Ethische Commissie Dierproeven, Ghent University, Ghent, Belgium).

\section{References}

1. Edvardsen T, Urheim S, Skulstad H, Steine K, Ihlen H, Smiseth OA (2002) Quantification of left ventricular systolic function by tissue doppler echocardiography: added value of measuring preand postejection velocities in ischemic myocardium. Circulation 105:2071-2077

2. Heimdal A, Støylen A, Torp H, Skjaerpe T (1998) Real-time strain rate imaging of the left ventricle by ultrasound. J Am Soc Echocardiogr 11:1013-1019

3. Kanai H, Hasegawa H, Chubachi N (1997) Noninvasive evaluation of local myocardial thickening and its color-coded imaging. IEEE Trans Ultrason Ferroelectr Freq Control 44:752-768

4. D'hooge J, Heimdal A, Jamal F, Kukulski T, Bijnens B, Rademakers F et al (2000) Regional strain and strain rate measurements by cardiac ultrasound: principles, implementation and limitations. Eur J Echocardiogr 1:154-170 
5. Sutherland GR, Di Salvo G, Claus P, D'hooge J, Bijnens B (2004) Strain and strain rate imaging: a new clinical approach to quantifying regional myocardial function. J Am Soc Echocardiogr 17:788-802

6. Luis SA, Yamada A, Khandheria BK, Speranza V, Benjamin A, Ischenko $\mathrm{M}$ et al (2014) Use of three-dimensional speckle-tracking echocardiography for quantitative assessment of global left ventricular function: a comparative study to three-dimensional echocardiography. J Am Soc Echocardiogr 27:285-291

7. Reant P, Barbot L, Touche C, Dijos M, Arsac F, Pillois X et al (2012) Evaluation of global left ventricular systolic function using three-dimensional echocardiography speckle-tracking strain parameters. J Am Soc Echocardiogr 25:68-79

8. Crosby J, Amundsen BH, Hergum T, Remme EW, Langeland S, Torp H (2009) 3-D speckle tracking for assessment of regional left ventricular function. Ultrasound Med Biol 35:458-471

9. Elen A, Choi HF, Loeckx D, Gao H, Claus P, Suetens P et al (2008) Three-dimensional cardiac strain estimation using spatiotemporal elastic registration of ultrasound images: a feasibility study. IEEE Trans Med Imaging 27:1580-1591

10. Nesser H-J, Winter S (2009) Speckle tracking in the evaluation of left ventricular dyssynchrony. Echocardiography 26:324-336

11. Kleijn SA, Aly MFA, Terwee CB, van Rossum AC, Kamp O (2011) Three-dimensional speckle tracking echocardiography for automatic assessment of global and regional left ventricular function based on area strain. J Am Soc Echocardiogr 24:314-321

12. Seo Y, Ishizu T, Enomoto Y, Sugimori H, Yamamoto M, Machino $\mathrm{T}$ et al (2009) Validation of 3-dimensional speckle tracking imaging to quantify regional myocardial deformation. Circ Cardiovasc Imaging 2:451-459

13. Jasaityte R, Heyde B, D'hooge J (2013) Current state of threedimensional myocardial strain estimation using echocardiography. J Am Soc Echocardiogr 26:15-28

14. Badano LP, Cucchini U, Muraru D, Al Nono O, Sarais C, Iliceto $\mathrm{S}$ (2013) Use of three-dimensional speckle tracking to assess left ventricular myocardial mechanics: inter-vendor consistency and reproducibility of strain measurements. Eur Heart J Cardiovasc Imaging 14:285-293

15. Farsalinos KE, Daraban AM, Ünlü S, Thomas JD, Badano LP, Voigt JU (2015) Head-to-Head comparison of global longitudinal strain measurements among nine different vendors: the EACVI/ ASE inter-vendor comparison study. J Am Soc Echocardiogr 28:1171-1181

16. National Research Council (US) Committee for the Update of the Guide for the Care and Use of Laboratory Animals (2011) Guide for the care and use of laboratory animals, 8th edn. National Academies Press, Washington (DC)

17. Heyde B, Bouchez S, Thieren S, Vandenheuvel M, Jasaityte R, Barbosa D et al (2013) Elastic image registration to quantify 3-D regional myocardial deformation from volumetric ultrasound: experimental validation in an animal model. Ultrasound Med Biol 39:1688-1697

18. Theroux P, Ross J, Franklin D, Kemper WS, Sasyama S (1976) Regional myocardial function in the conscious dog during acute coronary occlusion and responses to morphine, propranolol, nitroglycerin, and lidocaine. Circulation 53:302-314

19. Cerqueira MD, Weissman NJ, Dilsizian V, Jacobs AK (2002) Standardized Myocardial segmentation and nomenclature for tomographic imaging of the heart: a statement for healthcare professionals from the cardiac imaging committee of the council on clinical cardiology of the American Heart Association. Circulation 105:539-542

20. Pérez de Isla L, Balcones DV, Fernández-Golfín C, MarcosAlberca P, Almería C, Rodrigo JL et al (2009) Three-dimensional-wall motion tracking: a new and faster tool for myocardial strain assessment: comparison with two-dimensional-wall motion tracking. J Am Soc Echocardiogr 22:325-330

21. Maffessanti F, Nesser H-J, Weinert L, Steringer-Mascherbauer R, Niel J, Gorissen W et al (2009) Quantitative evaluation of regional left ventricular function using three-dimensional speckle tracking echocardiography in patients with and without heart disease. Am J Cardiol 104:1755-1762

22. Duan Q, Parker KM, Lorsakul A, Angelini ED, Hyodo E, Homma S et al (2009) Quantitative validation of optical flow based myocardial strain measures using sonomicrometry. Proc IEEE Int Symp Biomed Imaging 2009:454-457

23. Langeland S, Wouters PF, Claus P, Leather HA, Bijnens B, Sutherland GR et al (2006) Experimental assessment of a new research tool for the estimation of two-dimensional myocardial strain. Ultrasound Med Biol 32:1509-1513

24. Seo Y, Ishizu T, Enomoto Y, Sugimori H, Aonuma K (2011) Endocardial Surface area tracking for assessment of regional lv wall deformation with 3D speckle tracking imaging. JACC Cardiovase Imaging 4:358-365

25. Langeland S, D'hooge J, Wouters PF, Leather HA, Claus P, Bijnens B et al (2005) Experimental validation of a new ultrasound method for the simultaneous assessment of radial and longitudinal myocardial deformation independent of insonation angle. Circulation 112:2157-2162

26. Rösner A, Barbosa D, Aaersæther E, Kjønås D, Schirmer H, D'hooge J (2015) The influence of frame rate on two-dimensional speckle-tracking strain measurements: a study on silico-simulated models and images recorded in patients. Eur Heart J Cardiovasc Imaging 16:1137-1147

27. Gayat E, Ahmad H, Weinert L, Lang RM, Mor-Avi V (2011) Reproducibility and inter-vendor variability of left ventricular deformation measurements by three-dimensional speckle-tracking echocardiography. J Am Soc Echocardiogr 24:878-885

28. Voigt J-U, Pedrizzetti G, Lysyansky P, Marwick TH, Houle H, Baumann R et al (2015) Definitions for a common standard for 2D speckle tracking echocardiography: consensus document of the EACVI/ASE/Industry task force to standardize deformation imaging. J Am Soc Echocardiogr 28:183-193

29. Yang L, Georgescu B, Zheng Y, Wang Y, Meer P, Comaniciu D (2011) Prediction based collaborative trackers (PCT): a robust and accurate approach toward 3D medical object tracking. IEEE Trans Med Imaging 30:1921-1932

30. Cikes M, Tong L, Sutherland GR, D'hooge J (2014) Ultrafast cardiac ultrasound imaging: technical principles, applications, and clinical benefits. JACC Cardiovasc Imaging 7:812-823

31. Hjertaas JJ, Fosså H, Dybdahl GL, Grüner R, Lunde P, Matre K (2013) Accuracy of real-time single- and multi-beat 3-d speckle tracking echocardiography in vitro. Ultrasound Med Biol 39:1006-1014

32. Muraru D, Badano LP (2014) Quantitative analysis of the left ventricle by echocardiography in daily practice: as simple as possible, but not simpler. J Am Soc Echocardiogr 27:1025-1028 